Once you have Acrobat Reader open on your computer, click on the Comment tab at the right of the toolbar:

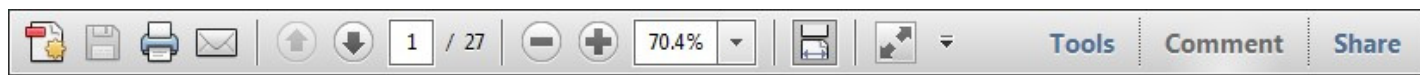

This will open up a panel down the right side of the document. The majority of tools you will use for annotating your proof will be in the Annotations section, pictured opposite. We've picked out some of these tools below:

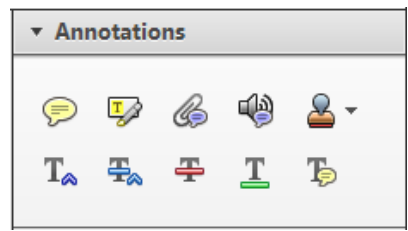

1. Replace (Ins) Tool - for replacing text.

Tistikes a line through text and opens up a text box where replacement text can be entered.

How to use it

- Highlight a word or sentence.

- Click on the Replace (Ins) icon in the Annotations section.

- Type the replacement text into the blue box that appears.

ıdard tramework for the analysis of $\mathrm{m}$ icy-Nevertheless, it also led to exog،

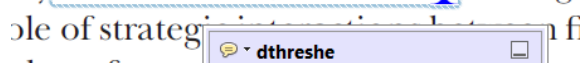
aber of comp 08/06/2011 15:58:17 $\quad$ O is that the $\mathrm{s} 1$, which led of nain compo: be level, are exc nc

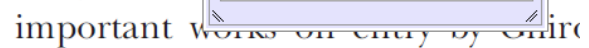
M heneforth) 1 we onen the 'hlarle $\mathrm{t}$

3. Add note to text Tool - for highlighting a section to be changed to bold or italic.

T) Highlights text in yellow and opens up a text box where comments can be entered.

How to use it

- Highlight the relevant section of text.

- Click on the Add note to text icon in the Annotations section.

- Type instruction on what should be changed regarding the text into the yellow box that appears.

namic responses of mark ups ent with the VAR evidence

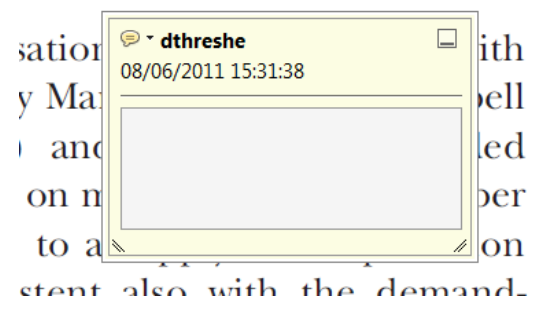

2. Strikethrough (Del) Tool - for deleting text.

T Strikes a red line through text that is to be deleted.

How to use it

- Highlight a word or sentence.

- Click on the Strikethrough (Del) icon in the Annotations section.

there is no room for extra protits al s ups are zero and the number of zet) values are not determined by Blanchard and Kiyotaki (1987), sfect competition in general equilil ts of aggregate demand and supply lassical framework assuming monol een on evorenous number of firme

4. Add sticky note Tool - for making notes at specific points in the text.

Marks a point in the proof where a comment needs to be highlighted.

How to use it

- Click on the Add sticky note icon in the Annotations section.

- Click at the point in the proof where the comment should be inserted.

- Type the comment into the yellow box that appears.

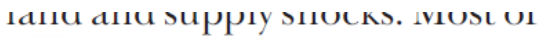

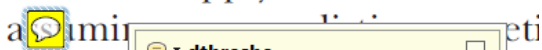

numbe $\mid \begin{aligned} & \ominus \text {-dthreshe } \\ & 08 / 06 / 2011 \text { 15:18:08 }\end{aligned}$

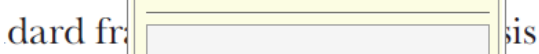

cy. Nev pa

le of st $\square$ wi

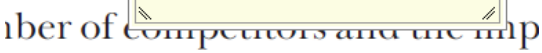

is that the structure of the secto 
5. Attach File Tool - for inserting large amounts of text or replacement figures.

Inserts an icon linking to the attached file in the appropriate place in the text.

\section{How to use it}

- Click on the Attach File icon in the Annotations section.

- Click on the proof to where you'd like the attached file to be linked.

- Select the file to be attached from your computer or network.

- Select the colour and type of icon that will appear in the proof. Click OK.

\section{E N D}

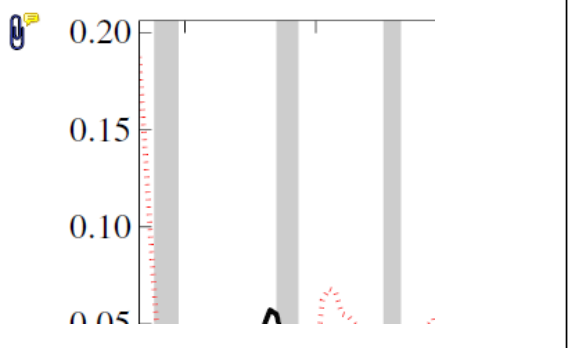

6. Drawing Markups Tools - for drawing shapes, lines and freeform annotations on proofs and commenting on these marks. Allows shapes, lines and freeform annotations to be drawn on proofs and for comment to be made on these marks.

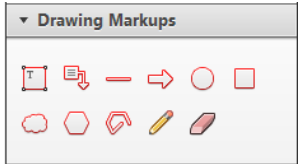

How to use it

- Click on one of the shapes in the Drawing Markups section.

- Click on the proof at the relevant point and draw the selected shape with the cursor.

- To add a comment to the drawn shape, move the cursor over the shape until an arrowhead appears.

- Double click on the shape and type any text in the red box that appears.

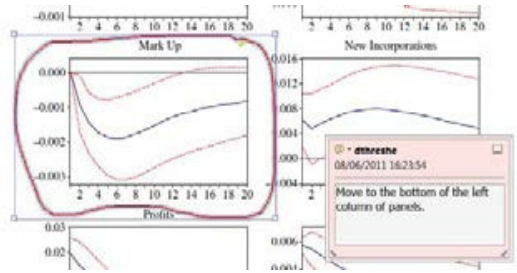




\begin{tabular}{|l|c|c|c|l|l|}
\hline \multirow{2}{*}{$\mathcal{C}$} & O P O & 12301 & \multirow{2}{*}{ WILEY } & Dispatch: 18.4.16 & CE: Jenifar Seles S \\
\cline { 2 - 3 } \cline { 5 - 6 } & Journal Code & Manuscript No. of pages: 7 & PE: Sakthivel R \\
\hline
\end{tabular}

\title{
The relationship between dioptric power and magnification in progressive addition lenses
}

\author{
1 Sergio Barbero and Javier Portilla \\ 2 Instituto de Óptica (CSIC), Madrid, Spain
}

Citation information: Barbero S, Portilla J. The relationship between dioptric power and magnification in progressive addition lenses. Ophthalmic Physiol Opt 2016. doi: 10.1111/opo.12301

Keywords: dioptric blurring, dioptric matrix, magnification, progressive addition lenses

Correspondence: Sergio Barbero

E-mail address: sergio.barbero@csic.es

Received: 12 February 2016; Accepted: 7 April 2016

\section{Introduction}

Progressive addition lenses (PALs) are a common solution for presbyopic eyes and include different refractive prescriptions for far, near and intermediate vision. In a PAL the different power required by the eye, when looking at distinct object locations, is supplied by an optical surface whose mean curvature changes gradually along a region

\begin{abstract}
Purpose: Non-uniform magnification (distortion) and dioptric blur are two major undesired optical defects affecting vision when looking through progressive addition lenses (PALs) and studying them is potentially very valuable for PAL design. The major purpose of this paper is to analyse the relationship between dioptric power and magnification and, additionally, to evaluate the expected values of distortion and dioptric blur typically present in PALs. This has not been carefully and rigorously analysed to date.

Methods: We computed the local dioptric and magnification matrices (using a two-ray differential method) for different gaze directions in an archetypical model of a PAL. We used four different maps: scalar magnification and anamorphic distortion, to describe magnification, and mean power and astigmatism, to describe dioptric power.

Results: There is a good correlation between scalar magnification and mean power on the one hand, and anamorphic distortion and astigmatism on the other hand. Changes of $1 \mathrm{D}$ in mean power are associated with variations in scalar magnification of around 3\%. Also, 3\% of anamorphic distortion is associated with increasing astigmatism up to $1 \mathrm{D}$. The directions of maximal power and maximal magnification are quite similar, though not equal (differences up to $\pm 1.5^{\circ}$ ). These directions strongly change from close to isotropic at the intermediate corridor to around $45^{\circ}$ of oblique inclination for a few degrees of horizontal eye rotation. In typical PALs the level of distortion (non-uniform magnification), which is unavoidably present when dioptric blurring appears, is small for usable vision zones (below $1 \mathrm{D}$ of astigmatism).

Conclusion: The combined analysis of dioptric power and magnification in PALs helps to understand their limitations as a visual aid. On the one hand, the potentials of including distortion magnitude as a target in the metric function being optimised in the PAL design are reduced; on the other hand, it seems worthwhile

to explore adding the degradation orientation as an additional target.
\end{abstract}

called the intermediate vision corridor. Around this area the PAL unavoidably introduces some undesired optical effects: optical blurring (principally astigmatism), nonuniform magnification (distortion) and image displacement. The latter is due to prismatic error, i.e. the direction change of the line of sight when viewing an object point through the lenses. Although image displacement has been reported to affect visual experience, for instance changing 
the vestibulo-ocular reflex, ${ }^{1}$ it will not be analysed in this paper.

Although the visual system is more tolerant to distortion than to blur (thanks to neural adaptation ${ }^{2}$ ), its relevance has not yet been completely assessed. It has been reported that visual alterations due to distortion are behind some cases of PAL wearer discomfort, ${ }^{3}$ specifically through alterations of space perception. These effects are more harmful in dynamic than in static vision, because distortion may affect postural balance ${ }^{4}$ or depth perception cues such as motion parallax. ${ }^{5}$ Additionally, magnification changes may explain the mobility problems associated with dynamic vision in some older adults, which occasionally can cause falls. ${ }^{6}$ Furthermore differences in magnification between the eyes can affect binocular vision. ${ }^{7}$

These concerns have been raised by some ophthalmic companies who have launched PAL designs tackling distortion. For instance, the Varilux S Series ${ }^{\mathrm{TM}}$ lens (launched by Essilor S.A.) was designed with the minimisation of anamorphic distortion as a goal ${ }^{8}$; or patent EP0809126 where the design strategy is based on a metric considering the difference in the mean magnification between the near and far portions. ${ }^{9}$

However, unfortunately little information can be found in the scientific literature about the amount of distortion induced by PALs and its influence on vision. As identified many years ago by Ogle in his classic Binocular Vision, ${ }^{7}$ there are few empirical studies on the tolerance to distortion induced by ophthalmic lenses partly because it is very difficult to quantify the tolerance to distortion isolated from blurring: if one is present, so is the other. In order to overcome this empirical limitation our approach in this paper takes full advantage of computer simulations to make a systematic study of the relationship between magnification and dioptric power, as well as to evaluate the expected values of distortion typically present in PALs.

We used methodology developed in a previous work by ourselves, ${ }^{10}$ in which magnification and first order defocus are locally characterised for each gaze direction by using the dioptric and magnification matrices. From them, four magnitudes are used to quantify dioptric blur and distortion: mean power vs magnification, and astigmatism vs anamorphic distortion, all of them as functions of eye rotation angles. We stress the fact that the power magnitudes measured with our method are those considering the wearer's point of view and the whole geometry of the lenses, as opposed to the surface power maps conventionally used in the field. We recommend references ${ }^{11,12}$ for a detailed discussion of this issue. Additionally, defocus blur and magnification ellipses (the latter were already proposed by $\mathrm{Ogle}^{7}$ as a visualisation tool) are used here to display the directions of maximum and minimum dioptric blurring and magnification for each gaze direction.

\section{Archetypical model of a progressive addition lens}

Sheedy et al. ${ }^{13}$ argued that the so-called Alvarez surface is an archetypical model of a progressive power surface:

$u(x, y)=A\left(\frac{y^{3}}{3}+y x^{2}\right)$

because it represents a surface containing an umbilical line $(y=0)$ where astigmatism is zero and where the power changes linearly in $y$. Following Sheedy we have adopted the Alvarez surface in our PAL model, though not without two warnings. First, modern PAL designs do not necessarily contain an umbilical line; vision tolerates some amount of cylinder, so relaxing the condition of zero astigmatism at the corridor line helps reduce the astigmatism in its surroundings. Second, as pointed out by Rubinstein, ${ }^{14}$ the Alvarez surface power linear dependence is referred to a local coordinate system (the $\mathrm{z}$ coordinate points along the normal to the surface) in the corridor line. Rubinstein ${ }^{14}$ also proved that the Minkwitz theorem does not hold in an absolute reference system for an Alvarez surface. Besides these two facts, any serious current PAL design involves free-form surface optimisation following sophisticated algorithms (see for instance ${ }^{15}$ and references there). Despite these concerns, the Alvarez surface is still an appropriate model, because any PAL surface is similar to an Alvarez surface close to the intermediate corridor. Furthermore, an Alvarez model, which induces unrealistic power errors when moving far from the corridor, is convenient for our purposes, because we are interested in evaluating the relationship between dioptric power and distortion for a wide range of values of both defects.

Our model comprises an Alvarez front progressive surface and a spherical back surface. We used a thin lens model. This does not mean that the effect of the non-progressive surface and the lens thickness is negligible, but just that this approximation is sufficient to model the relationship between magnification and dioptric power with different power ranges. Typical PALs have a front progressive lens -although some recent designs could have a progressive back surface ${ }^{16}$-and a spherical back surface called the base curve. The base curve substantially affects the optical performance of the PALs. ${ }^{12}$ Hence, we have explicitly analysed two different base curves. An extra parameter is required: the distance from the back vertex of the lens to the center of rotation of the eye, for which we assumed a typical value of $27 \mathrm{~mm}^{17}$

Menozzi et al. reported a tolerable range of $15^{\circ}$ departure from the most comfortable gaze direction in the vertical direction. ${ }^{18}$ Another study ${ }^{19}$ measured that for viewing objects located further than $7^{\circ}$ in the horizontal direction the head starts moving. Using this information we 
computed the dioptric and magnification matrices in an optical window of $\pm 7^{\circ}$ and from $0^{\circ}$ to $-15^{\circ}$ in the horizontal and vertical directions respectively.

When modelling dioptric power through PALs it is important to simulate the optical vergence associated to each object location. Here we follow a linear power law, i.e. the power changes linearly with the vertical coordinate of the PAL corridor. For each surface point the power equals the viewing object vergence. Therefore, and considering our PAL model, for each viewing object the vergence $\left(L_{o}\right)$ is calculated by obtaining the mean curvature of the Alvarez surface (Equation 1): Lo $(y)=2 A y(n-1)$, where $n$ is the refractive index of the lens and $A$ is the coefficient of Alvarez surface. Figure 1 illustrates this model.

\section{Computation of dioptric and magnification matrices}

Traditionally, power errors and magnification have been evaluated by using the paraxial approximation. Here we use the concept of rotational magnification matrix which evaluates the change in magnification associated with a line of sight when using spectacles with respect to not using them (see a full discussion elsewhere ${ }^{10}$ ). Within this framework, in rotationally symmetric ophthalmic lenses, there exists a formula relating the scalar spectacle magnification with the spectacle lens power ${ }^{10,20}:$ Nspec $=1 /$ $(1-\mathrm{dP}), \mathrm{d}$ being the distance from the spectacles to the rotation center of the eye and $\mathrm{P}$ being the optical power of the ophthalmic lens. However, this approximation is not valid for large eye rotations (note that when the gaze direction is not collinear to the normal of the surface at the intersection point the power changes), and neither for ophthalmic lenses without axial rotationally symmetry (rotation of principal directions). ${ }^{21} \mathrm{~A}$ PAL element is a clear example of it. For such cases a more sophisticated procedure is required. ${ }^{21}$

In this paper we computed dioptric and magnification matrices using a two-ray differential method proposed by us elsewhere. ${ }^{10}$ This method provides a more accurate computation as compared with the paraxial approximation. To

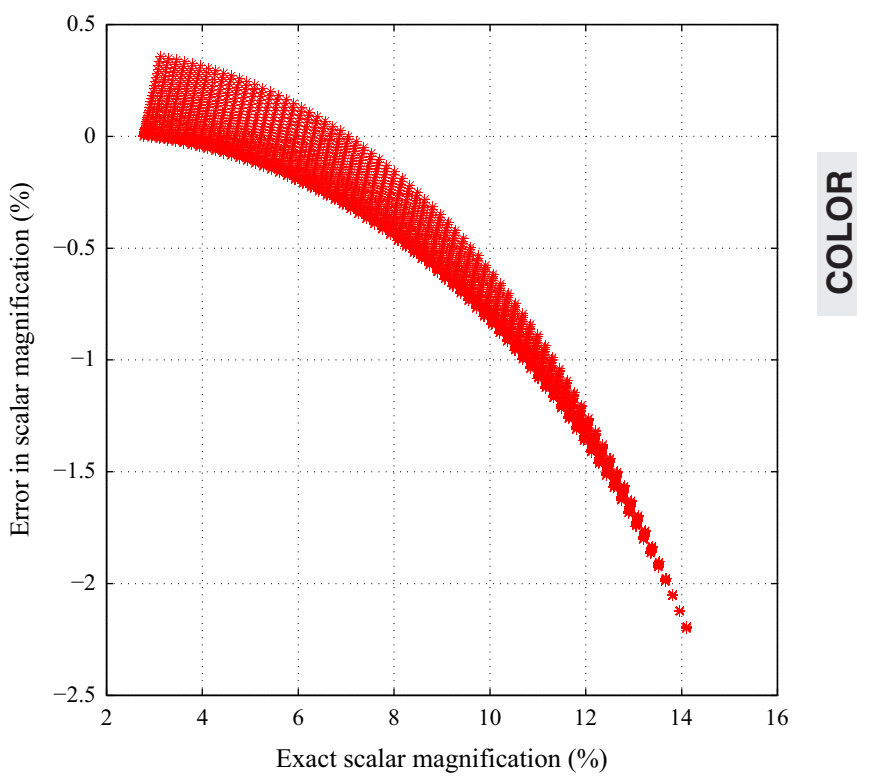

Figure 2. Errors in the scalar magnification (\%) when the paraxial.

illustrate this, Figure 2 shows the difference between the exact scalar magnification (percentage with respect to a reference value of 100\%) and one computed with the paraxial approximation for a test lens (test \#8 is explained in next section).

In this example we see that when the exact scalar magnification is $14 \%$ the approximated magnification value would be about $12 \%$; the absolute error can be up to $2.2 \%$ in the scalar magnification Errors in anamorphic magnification are expected to be higher.

Finally we note that, all through this paper, astigmatism means the difference between the two principal powers for each gaze direction.

\section{Tests}

We assumed that the material of the lenses was CR-39 (refractive index 1.499). We modelled lenses with $+1 \mathrm{D}$

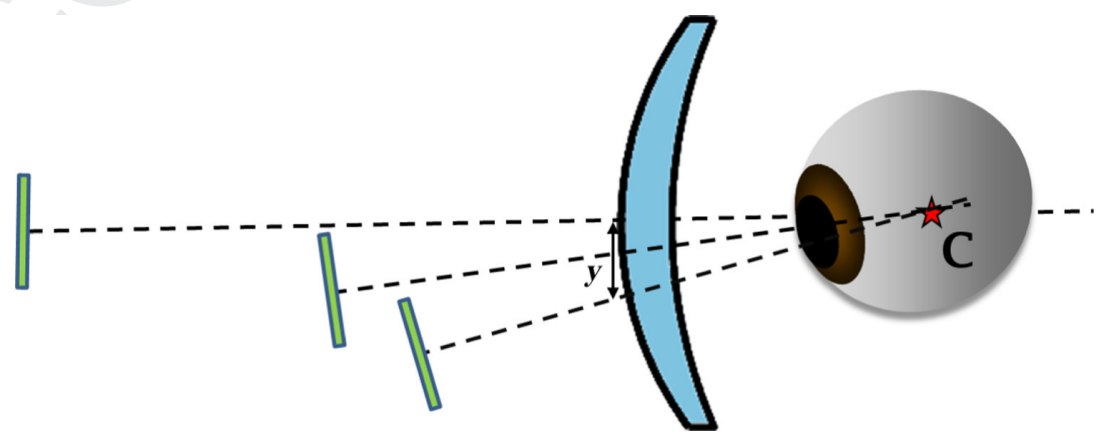

Figure 1. Object vergence changes linearly as a function of vertical distance (y) from primary line of sight (horizontal line). 
power for far vision, although any other far vision prescription could have been simulated. As mentioned above, the selection of the base curve is relevant to PAL performance. We used two different tests. First one (test \#2) uses a very flat base curve $(-2.0 \mathrm{D})$ and the other (test \#8) a very steep base curve $(-8.0 \mathrm{D}){ }^{12}$

Power addition in PALs rarely exceeds $3.5 \mathrm{D}$. Consequently, in order to analyse the complete range of powers that could be found in PALs we modelled an Alvarez surface with a maximum power around that value. This was attained when $A$ equals 0.0005 in the Alvarez equation (Equation 1).

\section{Results}

For tests \#2 and \#8, we evaluated the mean power vs the mean magnification and the astigmatism vs the anamorphic distortion, both as functions of eye rotations. Figure 3 shows mean power (Figure 3a), astigmatism (Figure 3b), scalar magnification (Figure $3 c$ ) and anamorphic distortion (Figure $3 d$ ) as functions of eye rotations for test \#2.

Mean power increases in a quasi-linear manner with vertical eye rotation. As predicted by Minkwitz theorem, ${ }^{13}$ the cylinder increases with eye rotation, almost double along the horizontal direction as compared to power variation along the vertical direction, specially along the $x=0$ curve.
As shown in Figures $3 b$, $d$, a similar relationship can be established between scalar magnification and anamorphic distortion.

Figure 4 shows similar results to those of Figure 3, now for test \#8. However it is worth noting that both scalar magnification and anamorphic distortion are slightly higher in test \#2 than in test \#8. This is in agreement with the fact that global distortion (in monofocal spectacles) is reduced when using steeper base curves. ${ }^{22}$

The correlation between the geometrical entities associated with the magnification matrix and those of the dioptric matrix are studied in more detail in Figure 5, for the example of test \#2. Blue dots represent the computed scalar and anamorphic magnification as function of mean power and astigmatism respectively. These data were fitted to a fourth order polynomial which is depicted as a red line.

We observe that there is a strong correlation between the scalar magnification and the mean power; $1 \mathrm{D}$ changes of mean optical power was associated with changes in scalar magnification of around 3\%.

A coarse correlation can also be established between astigmatism and anamorphic distortion with changes of $3 \%$ induced by cylinder errors up to $1 \mathrm{D}$. In reference ${ }^{10}$ we evaluated the relationship between astigmatism and anamorphic distortion in a highly asymmetric monofocal lens. It presented differences between measured and fitted

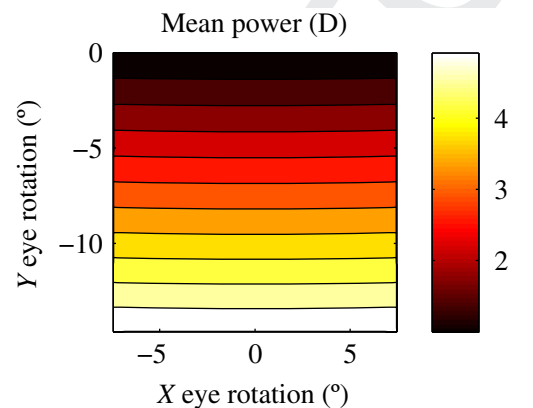

(a)

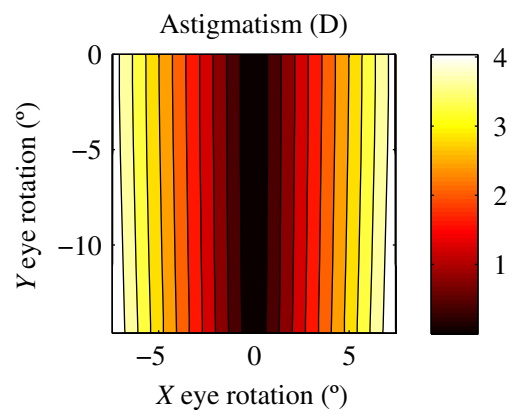

(b)

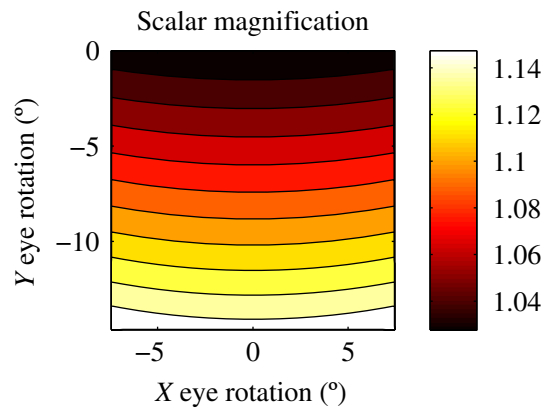

(c)

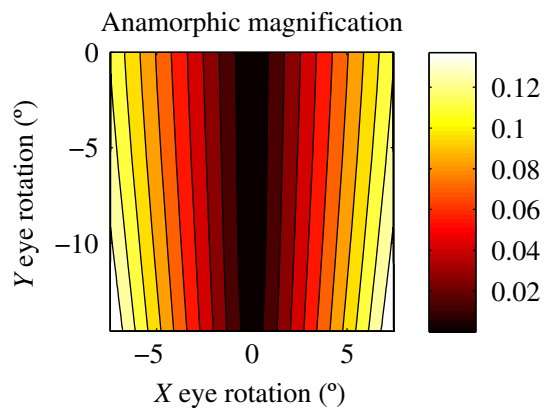

(d)

Figure 3. (a) Mean power (D) (b) Astigmatism (D) (c) Scalar magnification (d) Anamorphic distortion; as a function of eye rotations for test \#2. 


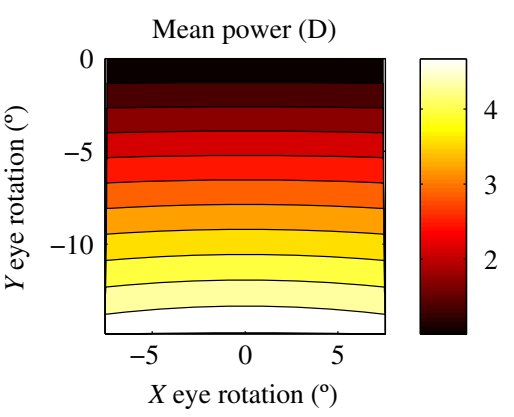

(a)

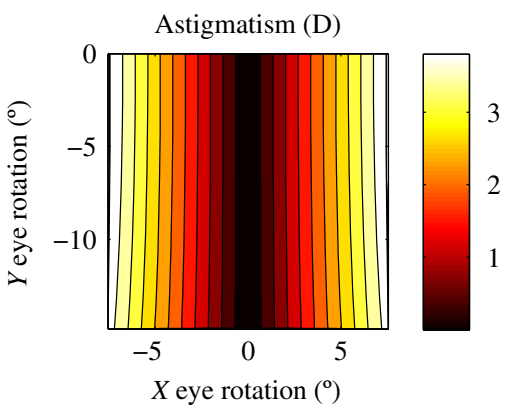

(b)

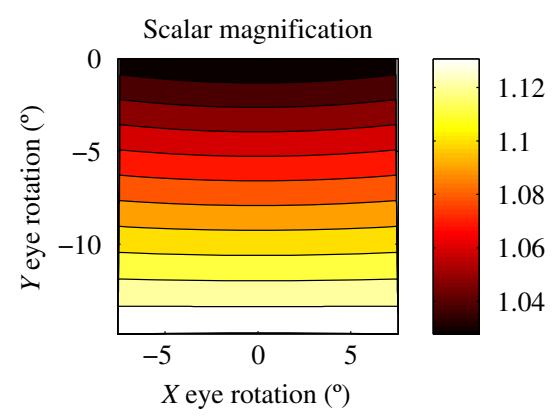

(c)

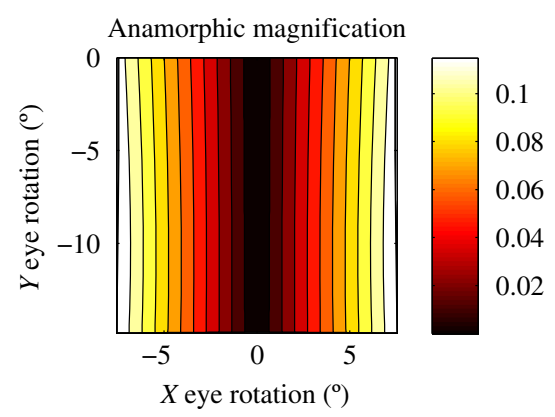

(d)

Figure 4. (a) Mean power (D) (b) Astigmatism (D) (c) Scalar magnification (d) Anamorphic distortion; as a function of eye rotations for test \#8.

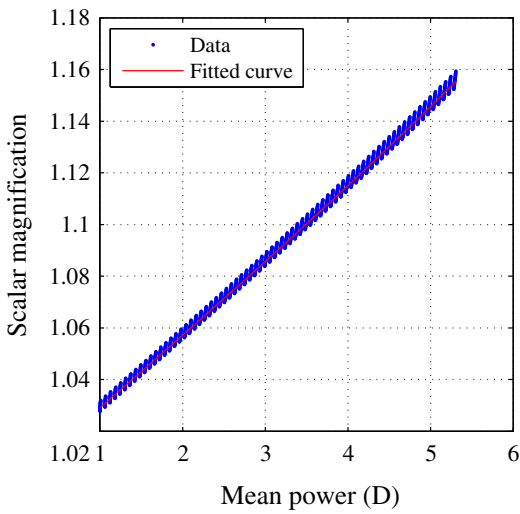

(a)

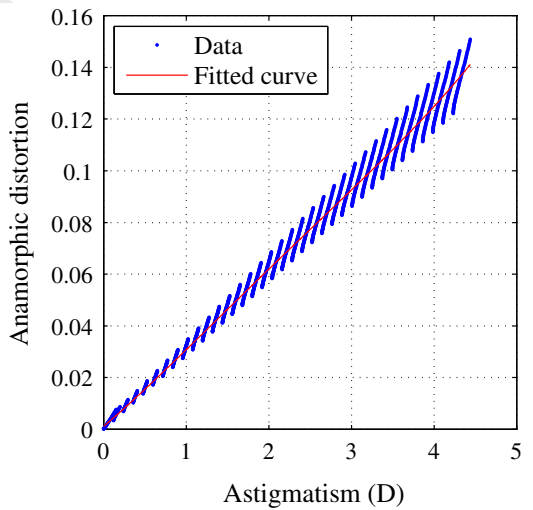

(b)

Figure 5. (a) Scalar magnification as function of mean power (D) (b) Anamorphic distortion as a function of astigmatism (D); both graphs for test \#2.

astigmatism up to $0.04 \mathrm{D}$. Here we find also differences up to $0.03 \mathrm{D}$. Additionally, as astigmatism increases, the dispersion of computed anamorphic distortion values with respect to the fitted quantities also increases. This fact is clearly seen in Figure 5b, but also indirectly in Figures $3 b, d$. Whereas iso-contours in the astigmatism map are essentially vertical lines, i.e. for a fixed horizontal eye rotation the cylinder is quite similar, this is not the case for the isocontours of the anamorphic distortion map, which tilts more from the vertical direction as the horizontal gaze direction increases. This behaviour can be explained by the following argument: the anamorphic distortion is more sensitive than astigmatism to changes in the angle formed between the incident ray and the normal of the surface at the incident point. For a fixed horizontal eye rotation this angle changes with the vertical eye rotation because of the cubic component of the PAL surface. As a consequence, the anamorphic distortion changes more rapidly than the astigmatism.

Finally we evaluated the relationship between the directions of maximal dioptric blurring and maximal magnification in PALs. An elliptical representation is shown in 


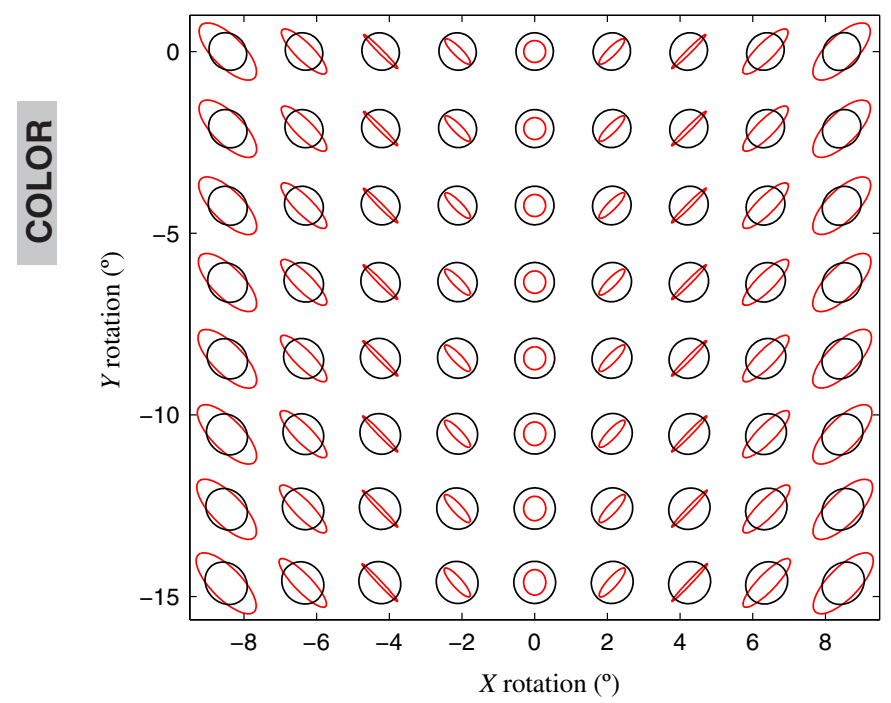

Figure 6. Elliptical representation of magnification (black ellipses) and dioptric blurring (red ellipses) for test \#2.

Figure 6. As seen in $^{10}$ the directions of maximal blurring and maximal magnification are quite similar, but not identical (differences up to $\pm 1.5^{\circ}$ ).

\section{Discussion}

Experimental studies with instant vision (not giving time for brain adaptation) have shown that an average eye may be able to discriminate $2-4 \%$ scalar magnification differences $^{7}$ (p. 231); including stereoscopic binocularity, the discrimination threshold could be reduced to $0.25 \%^{7}$ (p. 235). However, zones below $1 \mathrm{D}$ of astigmatism at the intermediate viewing area of the PAL are said to provide usable vision, ${ }^{23}$ which means that those parts of the lens which induce a higher astigmatic blur are not used by the eye because images seen through them are too degraded. Our simulations prove that distortion, due to both non-uniform scalar magnification and anamorphic distortion, reaches moderate values inside this $<1 \mathrm{D}$ astigmatism PAL area (below 3\%). Thus, distortion could be unnoticeable even without brain adaptation. This fact, in combination with the well-known better neural adaption to distortion as compared to blurring, would eventually explain why distortion is not as critical to PAL wearers as blurring. This conclusion is particularly relevant for PAL designers, because it somewhat lowers the expectations raised recently (as mentioned in the introduction) on the relative importance of distortion correction in PAL design.

However, we must warn that in this paper we have only analysed the local magnification associated with each gaze direction; that is, the distortion seen when we rotate our eye to look through different parts of the lens. If extra-foveal vision were to be included in the model, then global magnification, which provides a general description of the one-to-one mapping between ray direction incidents into the eye with and without spectacles (see full discussion elsewhere ${ }^{10}$ ), should be also considered.

The degradation orientation (direction of maximal degradation) both, for dioptric blurring and distortion, is also expected to be a relevant parameter in visual performance. Indeed, the role of blur orientation on visual performance has been recently addressed using adaptive optics. ${ }^{24}$ It has been shown that the loss of visual acuity due to induced astigmatism depends on the direction of maximal blurring. For example, for subjects without astigmatism, blurring in the vertical meridian is less harmful than in the horizontal one. ${ }^{24}$ However, to our knowledge, the same type of analysis concerning the visual impact of the direction of maximal distortion has not yet been tackled. As shown in this study, PALs strongly change the direction of maximum degradation, from close to isotropic at the intermediate corridor to around $\pm 45^{\circ}$ of oblique inclination for a few degrees of horizontal eye rotation. Considering all this, we think that the optical designer may consider including the degradation orientation as a goal in the metric function being optimised in PAL design.

\section{Acknowledgements}

This work was supported by grant FIS2012-30820.

\section{Disclosure}

The authors report no conflicts of interest.

\section{References}

1. Michaelides E \& Schutt CA. The correlation between the vestibulo-ocular reflex and multi-focal ocular correction: implications for vestibular compensation. Am J Otolaryngol 2014; 35: 572-576.

2. Adams WJ, Banks MS \& van Ee R. Adaptation to threedimensional distortions in human vision. Nat Neurosci 2001; 4: 1063-1064.

3. Gresset J, Fauquier C, Frenette B et al. Validation of a questionnaire on distortion perception among progressive addition lens wearers. In: Vision Science and its Applications, OSA Technical Digest 2000, paper MD2. https://www.osapublishing.org/abstract.cfm?uri=VSIA-2000-MD2, accessed $8 / 4 / 2016$

4. Faubert J \& Allard R. Effect of visual distortion on postural balance in a full immersion stereoscopic environment. In: Stereoscopic Displays and Virtual Reality, SPIE, 2004: 491500, http://proceedings.spiedigitallibrary.org/proceeding.aspx?articleid=836760, accessed 8/4/2016. 
5. Faubert J. The influence of optical distortions and tranverse chromatic aberration on motion parallax and stereopis in natural and artificial enviroments. In: Three-Dimensional Television, Video and Display Technologies (Javidi B \& Okano F, editors), Springer Verlag: New York, 2002; pp. 359-396.

6. Chapman GJ, Scally AJ \& Elliott DB. Adaptive gait changes in older people due to lens magnification. Ophthalmic Physiol Opt 2011; 31: 311-317.

7. Ogle KN. Researches in Binocular Vision, 1st edition, WB Saunders Company: Philadelphia, 1950.

8. Guilloux C, Rossi H, Marin Get al. The importance of the ophthalmic progressive lens shape on the space perception. In: Proceedings of the European Academy of Optometry and Optics; 2012; Dublin, Ireland, 20-22 April 2012.

9. Kazutoshi K \& Hiroyuki M. Progressive spectacle lens with progressive surface and correction of astigmatism provided on the rear side of the lens. European patent EP0809126, 2003.

10. Barbero S \& Portilla J. Geometrical interpretation of dioptric blurring and magnification in ophthalmic lenses. Opt Express 2015; 23: 13185-13199.

11. Arroyo R, Crespo D \& Alonso J. Scoring of progressive power lenses by means of user power maps. Optom Vis Sci 2012; 89: E489-E501.

12. Arroyo R, Crespo D \& Alonso J. Influence of the Base Curve in the Performance of Customized and Classical Progressive Lenses. Optom Vis Sci 2013; 90: 282-292.

13. Sheedy JE, Campbell C, King-Smith E \& Hayes JR. Progressive Powered Lenses: the Minkwitz Theorem. Optom Vis Sci 2005; 82: 916-922.
14. Rubinstein J. On the relation between power and astigmatism near an umbilic line. J Opt Soc Am A 2011; 28: 734-737.

15. Zhou F. Design of progressive additional lens with wavefront tracing method. $\mathrm{PhD}$, University of Minnesota, 2010. http://gradworks.umi.com/34/33/3433002.html, accessed 8/ 4/2016.

16. Qin L, Qian L \& Yu J. Design and fabrication of the progressive addition lenses. Proc. SPIE 8202 2011: 820215-1-6. http://proceedings.spiedigitallibrary.org/proceeding.aspx?articleid $=1195211$ \&resultClick $=1$

17. Atchison DA. Modern optical design assessment and spectacle lenses. Opt Acta (Lond) 1985; 32: 607-634.

18. Menozzi M, Vonbuol A, Krueger H \& Miege C. Direction of gaze and comfort: discovering the relation for the ergonomic optimization of visual tasks. Ophthalmic Physiol Opt 1994; 14: 393-399.

19. Sheedy JE \& Hardy RF. The optics of occupational progressive lenses. Optometry 2005; 76: 432-441.

20. Keating MP. Geometric, Physical, and Visual Optics, 2nd edition, Butterworth-Heinemann: Boston, 2002.

21. Stavroudis ON. The Mathematics of Geometrical and Physical Optics: The k-Function and its Ramifications, 1st edition, Wiley-VCH: Mörlenbach, 2006.

22. Fowler C \& Latham Petre K. Spectacle Lenses: Theory and Practice, 1st edition, Butterworth-Heinemann: Oxford, 2001.

23. Meister DJ \& Fisher SW. Progress in the spectacle correction of presbyopia. Part 1: Design and development of progressive lenses. Clin Exp Optom 2008; 91: 240-250.

24. Vinas M, de Gracia P, Dorronsoro C et al. Astigmatism impact on visual performance: Meridional and adaptational effects. Optom Vis Sci 2013; 90: 1430-1442. 


\section{Author Query Form}

Journal: $\quad$ OPO

Article: 12301

Dear Author,

During the copy-editing of your paper, the following queries arose. Please respond to these by marking up your proofs with the necessary changes/additions. Please write your answers on the query sheet if there is insufficient space on the page proofs. Please write clearly and follow the conventions shown on the attached corrections sheet. If returning the proof by fax do not write too close to the paper's edge. Please remember that illegible mark-ups may delay publication.

Many thanks for your assistance.

\begin{tabular}{|l|l|}
\hline Query reference & Query \\
\hline 1 & $\begin{array}{l}\text { AUTHOR: Please confirm that given names (red) and surnames/family names } \\
\text { (green) have been identified correctly. }\end{array}$ \\
\hline 2 & $\begin{array}{l}\text { AUTHOR: Please check that authors and their affiliations are correct. } \\
30820 " .\end{array}$ \\
\hline 3 & purovide funding agency for this funding number "FIS2012- \\
\hline
\end{tabular}

Remarks 